\title{
A High Throughput Assay for Discovery of Bacterial $\beta$-Glucuronidase Inhibitors
}

\author{
Syed Ahmad ${ }^{1}$, Mark A. Hughes ${ }^{1}$, Kimberly T. Lane ${ }^{2}$, Matthew R. Redinbo ${ }^{2,3,4}$, Li-An Yeh ${ }^{1}$ and \\ John E. Scott*,1
}

${ }^{1}$ Department of Pharmaceutical Sciences, Biomanufacturing Research Institute and Technology Enterprise, North
Carolina Central University, 1801 Fayetteville Street, Durham, NC 27707, USA
${ }^{2}$ Department of Chemistry, ${ }^{3}$ Department of Biochemistry and Biophysics, ${ }^{4}$ Lineberger Comprehensive Cancer Center,
University of North Carolina at Chapel Hill, Chapel Hill, North Carolina 27599 , USA

\begin{abstract}
CPT-11 is a widely-used anti-cancer drug that is converted in vivo to its active metabolite, SN-38. In the liver, enzymes detoxify SN-38 by coupling it to a glucuronidate moiety and this inactive compound (SN-38G) is excreted into the gastrointestinal tract. In the intestine, commensal bacteria convert the SN-38G back to the active and toxic SN-38 using bacterial $\beta$-glucuronidase enzyme (GUS). This intestinal SN-38 causes debilitating diarrhea that prevents doseintensification and efficacy in a significant fraction of patients undergoing CPT-11 treatment for cancer. This CPT-11 metabolic pathway suggests that small molecule inhibitors of GUS may have utility as novel therapeutics for prevention of dose-limiting diarrhea resulting from CPT-11 therapy. To identify chemical inhibitors of GUS activity, we employed and validated a high throughput, fluorescence-based biochemical assay and used this assay to screen a compound library. Novel inhibitors of GUS were identified with $\mathrm{IC}_{50}$ values ranging from $50 \mathrm{nM}$ to $4.8 \mu \mathrm{M}$. These compounds may be useful as chemical probes for use in proof-of-concept experiments designed to determine the efficacy of GUS inhibitors in altering the intestinal metabolism of drugs. Our results demonstrate that this high throughput assay can be used to identify small molecule inhibitors of GUS.
\end{abstract}

Keywords: $\beta$-glucuronidase, CPT-11, screen, inhibitor.

\section{INTRODUCTION}

Camptothecin is a potent anti-cancer compound that was originally purified from the tree Camptotheca acuminata and whose structure was elucidated in 1966 [1]. It inhibits the catalytic cycle of human topoisomerase I, which regulates the superhelical tension associated with DNA replication and is preferentially active in rapidly proliferating cells $[2,3]$. In preliminary human studies, camptothecin exhibited significant toxicity and poor bioavailability [4]. The camptothecin derivatives topotecan and CPT-11 (also called irinotecan) have since been discovered to have improved toxicity and bioavailability properties and these drugs are now in clinical use as anti-cancer therapies [4], although they still generate debilitating side effects in cancer patients that limit efficacy. CPT-11 is one of the three commonly-used chemotherapeutic agents for colon, lung, and brain cancer and it has also been used against refractory forms of leukemia and lymphoma [5]. CPT-11 is a prodrug, having a carbamate-linked dipiperidino group that significantly increases its solubility and bioavailability [4]. This dipiperidino group is removed in vivo by carboxylesterases to produce the active metabolite, $\mathrm{SN}-38$ [6].

The dose-limiting side effect of CPT-11 is severe diarrhea generated by its complex activation and metabolism

*Address correspondence to this author at 1801 Fayetteville St, BRITE Bldg, Rm 1019, North Carolina Central University, Durham, NC 27707, USA; Tel: 919-530-7569; Fax: 919-530-6600; E-mail: jscott@nccu.edu
[7, 8]. SN-38, the active metabolite of CPT-11, is glucuronidated in the liver by UDP-glucuronosyltransferase (UGT) enzymes [9] resulting in the inactive SN-38G [10], which is excreted via the biliary ducts into the gastrointestinal (GI) tract. Once in the intestines, $\mathrm{SN}-38 \mathrm{G}$ serves as a substrate for bacterial $\beta$-glucuronidase (GUS) enzymes that are produced by bacteria normally inhabiting the intestines. The removal of the glucuronide group by GUS generates a carbon source for the bacteria and, in the process $\mathrm{SN}-38 \mathrm{G}$ is reactivated back to the active and toxic SN-38 [11, 12]. This reactivated $\mathrm{SN}-38$, now present in the intestinal lumen, is toxic to the intestinal cells resulting in delayed diarrhea that prevents dose-intensification and efficacy in a significant fraction of patients undergoing CPT-11 treatment for cancer [13, 14].

The concept of using antibiotics to reduce GI bacteria levels to prevent the regeneration of $\mathrm{SN}-38$ in the intestine has been examined [15]. However, the use of antibiotics has several disadvantages. Intestinal bacteria function in essential pathways in carbohydrate metabolism, vitamin production, and the processing of bile acids, sterols and xenobiotics $[16,17]$. Thus, the removal of GI bacteria is not recommended for patients already challenged by cancer and chemotherapy. In addition, elimination of symbiotic GI flora increases the chances of infections by pathogenic bacteria, including enterohemorrhagic E. coli and Clostridium difficile [18-24].

$\beta$-glucuronidase enzymes hydrolyze glucuronic acid sugar moieties from a variety of compounds [25]. The pres- 
ence of GUS in a wide range of bacteria is exploited to detect bacterial contamination in commonly-used water purity tests [26]. Prior to our work, only relatively weak inhibitors of $\beta$-glucuronidases with $K_{i}$ values ranging from $25 \mu \mathrm{M}$ to 2 $\mathrm{mM}$ have been described $[27,28]$. Thus, we have sought to identify potent and selective inhibitors of bacterial $\beta$ glucuronidases to inhibit the generation of SN-38 in the intestines and thus reduce or eliminate the GI toxicity of CPT11 treatment without killing the useful bacteria required for intestinal health. From the high throughput screen for GUS inhibitors that is described in this report, we selected four hits for follow-up studies. We have recently published the results of these studies [29]. These compounds showed complete selectivity for bacterial GUS verses the homologous mammalian enzyme. The compounds demonstrated GUS inhibitory activity in living bacteria with $\mathrm{IC}_{50}$ values ranging from $18 \mathrm{nM}$ to $1.3 \mu \mathrm{M}$ with no effect on bacterial viability even at $100 \mu \mathrm{M}$. Furthermore, oral administration of one of these inhibitors protected mice from CPT-11-induced diarrhea.

In this report, we describe the development and validation of the high throughput GUS activity assay that led to the discovery of small molecule inhibitors of GUS activity. An existing GUS activity assay using the fluorigenic substrate 4methylumbelliferyl glucuronide (4MUG) was modified and formatted for HTS. In this assay, the non-fluorescent $4 \mathrm{MUG}$ is hydrolyzed by GUS generating 4-methylumbelliferone, a highly fluorescent molecule. This optimized and validated assay was then used to screen a chemical library in search of inhibitors of GUS.

\section{MATERIALS AND METHODS}

\section{Reagents}

All common reagents such as HEPES, Triton X-100, DGlucaric acid-1,4-lactone and dimethyl sulfoxide (DMSO) were reagent-grade quality obtained from Thermo Fisher Scientific (Waltham, MA) or Sigma-Aldrich (St. Louis, $\mathrm{MO})$. 4-methylumbelliferyl glucuronide (4MUG) was obtained from Sigma. The solid black 384-well plates (cat\# 3573) for the assay were from Corning Incorporated (Corning, NY). The Prestwick Chemical Library was obtained from Prestwick Chemical Company (Washington DC). Additional compounds for screening and $\mathrm{IC}_{50}$ determinations were obtained from the Asinex Corporation (Moscow, Russia). The expression and purification of the GUS enzyme from BL21-DE3 E. coli cells carrying an expression plasmid containing the full-length $E$. coli GUS gene has been previously described [29].

\section{GUS Assay}

The GUS assay was performed by the addition of $0.5 \mu \mathrm{l}$ of compound (or DMSO) to the well of a black 384-well plate followed by the addition of $30 \mu \mathrm{l}$ of diluted GUS enzyme (83 pM GUS). The enzyme was diluted into $50 \mathrm{mM}$ HEPES, pH 7.4 and $0.017 \%$ Triton X-100. After addition of enzyme, the reaction was initiated by addition of $20 \mu \mathrm{l}$ of 4MUG substrate $(312.5 \mu \mathrm{M} 4 \mathrm{MUG})$ diluted into $50 \mathrm{mM}$ HEPES, pH 7.4. 4MUG stock solutions were made in the same buffer. Final buffer in the assembled assay was $50 \mathrm{mM}$ HEPES, pH 7.4 and $0.01 \%$ Triton X-100. Following the initiation of the reaction with $4 \mathrm{MUG}$, the plates were incubated for 30 minutes at $23^{\circ} \mathrm{C}$ followed by the addition of $20 \mu \mathrm{l}$ of 1 $\mathrm{M}$ sodium carbonate solution to stop the reaction. The GUS, 4MUG, and stop solutions were delivered to the plate using Multidrop $^{\mathrm{TM}}$ liquid handlers (Thermo). The plate was then read in the Victor $\mathrm{V}$ plate reader. The fluorescence was measured using the $355 \mathrm{~nm}$ excitation filter and $460 \mathrm{~nm}$ emission filter (the ubelliferone filter set). Read time was 0.1 $\mathrm{s} /$ well and fluorescence expressed in relative fluorescence units (RFU). Error bars for data points represent standard deviations. Z'-factor values were derived by the published formula with $Z$ '-factors of $0.5-1.0$ indicating that an assay is robust for screening [30].

\section{High Throughput Screen}

For the high throughput screening of the Asinex chemical library, $0.5 \mu \mathrm{l}$ of $1 \mathrm{mM}$ compound in $100 \%$ DMSO was added to empty 384-well plates using a Biomek NX (Beckman Coulter Inc., Fullerton, CA), resulting in a final compound concentration of $10 \mu \mathrm{M}$ for the primary screen. This dry spotting of compounds into assay plates was performed as a routine method of conserving compound stock solutions. This spotting method has been validated for transfer of $0.5 \mu \mathrm{l}$ DMSO with a CV of $6.3 \%$ and $98 \%$ accuracy. Final assay concentrations in the $50 \mu \mathrm{l}$ enzyme reaction were $50 \mathrm{pM}$ GUS, $125 \mu \mathrm{M}$ 4MUG, $10 \mu \mathrm{M}$ compound, 1\% DMSO (from compound). For the screening, each plate had maximum $(\max )$ and minimum $(\min )$ controls with DMSO spotted in the wells. Max signal positive control wells contained only DMSO (no compound), while the min signal control wells were obtained by adding enzyme dilution buffer instead of GUS. D-Glucaric acid-1,4-lactone would not dissolve in DMSO or water at a high enough concentration to generate the min controls. The percent inhibition was calculated based on the max and min controls on the plate. The definition of a hit was a compound that displayed $\geq 50 \%$ inhibition in the primary screen. $\mathrm{IC}_{50}$ value was defined as the concentration of inhibitor calculated to inhibit $50 \%$ of the assay signal based on a serial dilution of compound. Values were calculated using either a four or three-parameter dose response (variable slope) equation in Graphpad Prism or ActivityBase (IDBS, Alameda, CA). For the $\mathrm{IC}_{50}$ determinations, serial dilutions of compounds were performed in $100 \%$ DMSO with a two-fold dilution scheme resulting in 10 concentrations of compound starting at a high concentration of $20 \mu \mathrm{M}$ compound in the final assay. For the HTS assay, these compound dilutions were spotted onto plates and the assay performed at $1 \%$ DMSO final concentration as described for the primary screen.

\section{RESULTS}

Bacterial GUS activity can readily be measured using the substrate 4-methylumbelliferyl glucuronide (4MUG) [26]. Upon hydrolysis of 4MUG by GUS, the fluorescent compound 4-methylumbelliferone (4MU) is generated. We used this substrate to detect GUS activity in an end point assay that is amenable for high throughput screening for inhibitors of GUS. The assay was developed with a final volume of 50 $\mu 1$ using the 384-well plate format to match the intended screening format. The general assay set-up included the addition of diluted GUS enzyme to the well followed by the addition of $4 \mathrm{MUG}$ to initiate the reaction (Fig. 1). The reaction was terminated with the addition of a $1 \mathrm{M} \mathrm{Na}_{2} \mathrm{CO}_{3}$ solution 
and the fluorescence measured in a plate reader. The final assay buffer consisted of $50 \mathrm{mM}$ HEPES pH 7.4 and $0.01 \%$ TX-100. The $\mathrm{K}_{\mathrm{m}}$ for $4 \mathrm{MUG}$ was experimentally determined to be $125 \mu \mathrm{M}$ (data not shown) and therefore this was the concentration of $4 \mathrm{MUG}$ used in this assay. Stopping of the reaction was confirmed by time course studies following termination of the assay (data not shown).

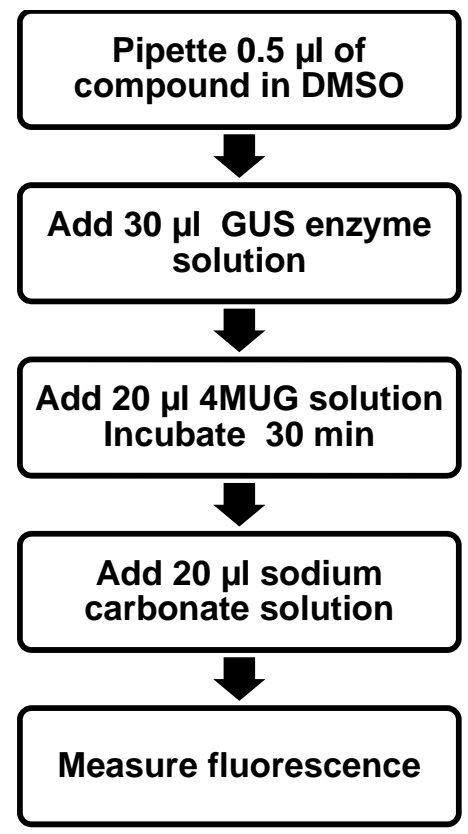

Fig. (1). Schematic of the GUS high throughput enzyme assay.

The activity in the assay was directly proportional to the concentration of GUS, up to at least 100 pM enzyme (Fig. 2). We chose to use $50 \mathrm{pM}$ as the final concentration in the assay to provide a robust assay window. This concentration of enzyme resulted in a linear time course in this assay for 40 minutes under final assay conditions in the presence of $1 \%$ DMSO (Fig. 3). The enzyme titration and time course data suggested that the assay conditions should be sensitive to compounds that are competitive inhibitors of GUS activity.

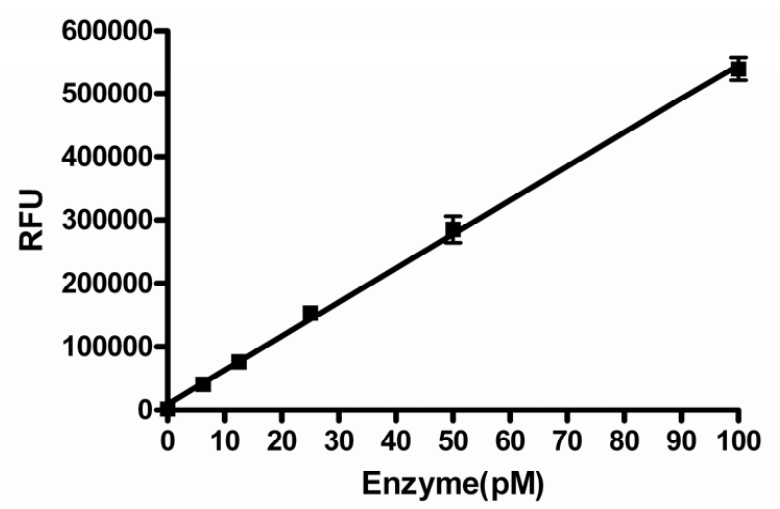

Fig. (2). GUS enzyme titration. GUS was titrated into the assay under final assay conditions with an incubation time of $30 \mathrm{~min}$ in the presence of $1 \%$ DMSO. Data points represent the average of three determinations per concentration and error bars represent standard deviations. Data are representative of three independent experiments.

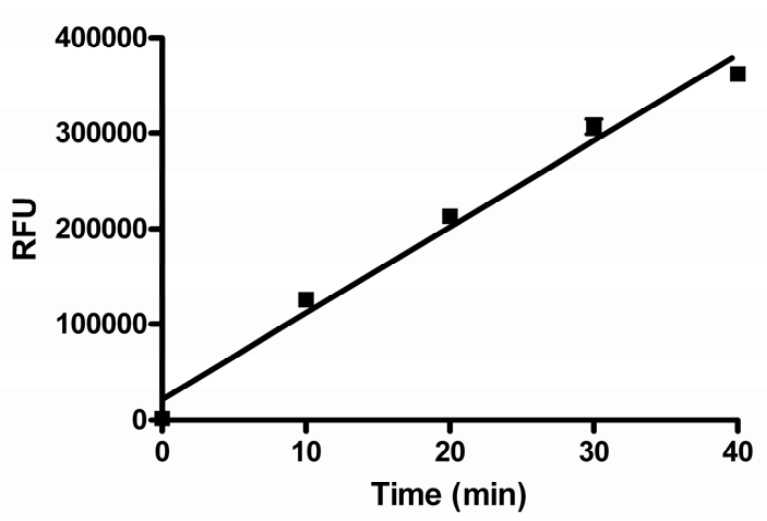

Fig. (3). GUS assay time course. A time course study was performed under final assay conditions in the presence of $1 \%$ DMSO. Data points represent the average of three determinations per time point and error bars represent standard deviations. Data are representative of three independent experiments.

Since compounds to be screened are dissolved in DMSO, the tolerance for DMSO was examined (Fig. 4). The assay was shown to be tolerant to DMSO concentrations up to $4 \%$. In the final screening assay, the maximal concentration that the enzyme is exposed to is $2 \%$ DMSO, before the addition of substrate, after which the final DMSO was set at 1\%. All experiments in this report were performed in the presence of $1 \%$ DMSO.

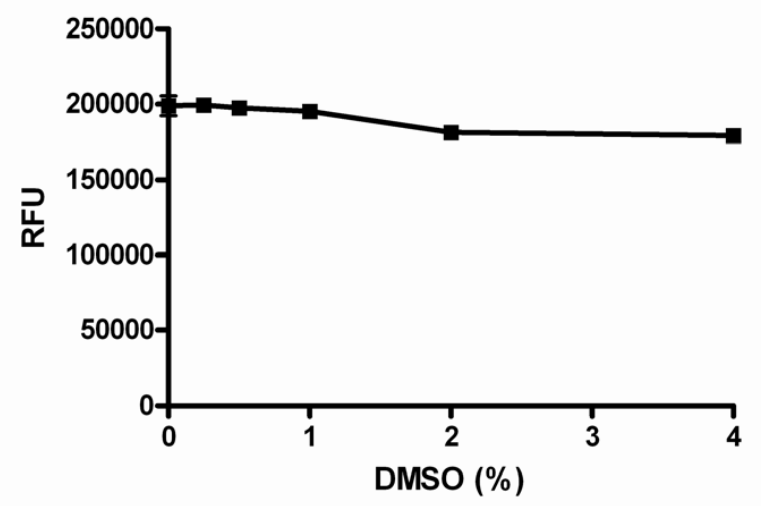

Fig. (4). DMSO tolerance of GUS assay. The indicated concentrations of DMSO were tested in the GUS assay. Data points represent the average of three determinations per concentration and error bars represent standard deviations. Data are representative of three independent experiments.

The variability of the assay was characterized in a 384well plate format. The HTS version of the assay employed a work-station level automated liquid handling system using the Biomek NX for delivery of compound or DMSO $(0.5 \mu 1)$ to the plate followed by addition of GUS $(30 \mu \mathrm{l}), 4 \mathrm{MUG}(20$ $\mu \mathrm{l})$ and $\mathrm{Na}_{2} \mathrm{CO}_{3}$ solution $(20 \mu \mathrm{l})$, in that order, using the Multidrop $^{\mathrm{TM}}$ liquid handler (Thermo). To assess single-point variability of the assay with full automation, all wells of a 384-well plate were pre-spotted with $0.5 \mu \mathrm{l}$ of DMSO (Fig. 5). The pre-spotted DMSO plates simulated compound plates 
that were to be screened. Two plates for each condition were used to determine the maximum (max) signal and minimum (min) signal. The $\% \mathrm{CV}$ of the max and min plates were 2.6 and $17.5 \%$, respectively. The $Z$ '-factor for the inhibition assay window was 0.81 . D-Glucaric acid-1,4-lactone has been reported to inhibit GUS [31]. The $\mathrm{IC}_{50}$ value for this control inhibitor was determined using the HTS protocol, including use of automation (Fig. 6). The $\mathrm{IC}_{50}$ values obtained were 21 and $17 \mu \mathrm{M}$ for two separate determinations. Solubility of this control inhibitor limited the use of concentrations $>100 \mu \mathrm{M}$, hence partial $\mathrm{IC}_{50}$ curves were obtained. Despite this limitation, these values are in good agreement with the published $K_{i}$ value of $25 \mu \mathrm{M}$ [31]. The Hill slopes were both 1.07 , very close to the expected value of 1.0 for inhibition of a single enzyme. Thus, reproducible $\mathrm{IC}_{50}$ values can be obtained using the automated version of the GUS assay. This data indicated that the assay was highly robust with minimal variability and amenable for high throughput screening for inhibitors of GUS activity.

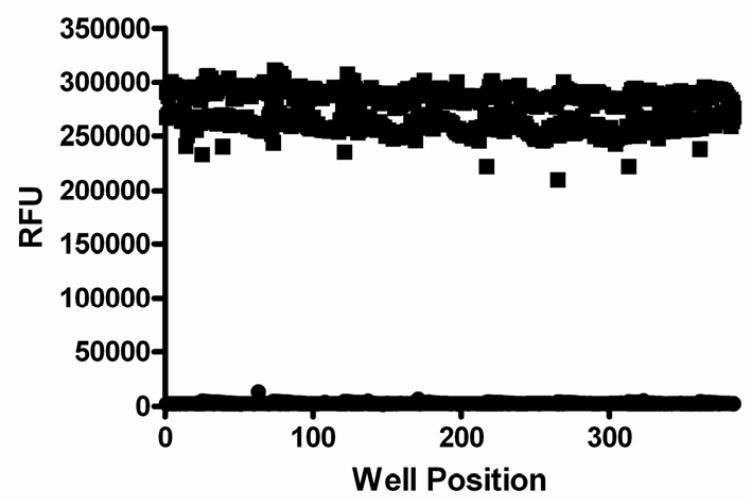

Fig. (5). GUS assay variability assessment. All wells of a 384well plate were pre-spotted with $0.5 \mu 1$ of DMSO. Two plates each were used to determine the maximum signal ( $\mathbf{a})$ and minimum signal $(\bullet)$.

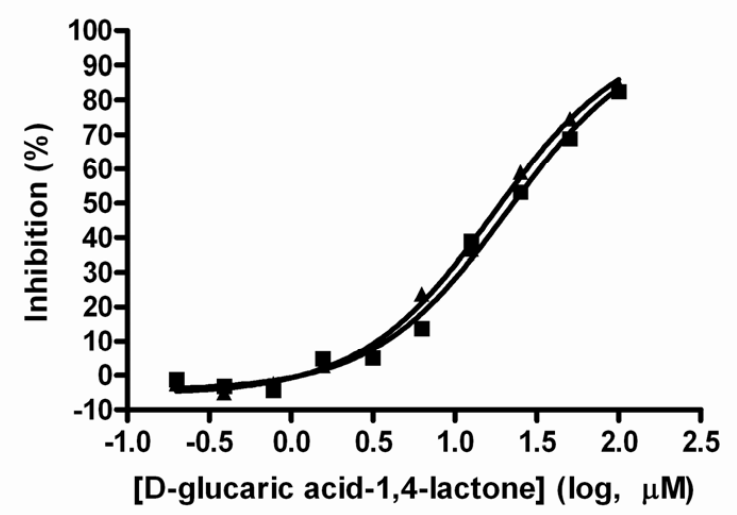

Fig. (6). $\mathrm{IC}_{50}$ value determinations using the automated GUS assay. Data points represent the average of three determinations per concentration and error bars represent standard deviations. Data are representative of two independent experiments.

As part of assay validation, a small collection of 1,120 compounds purchased from the Prestwick Chemical com- pany was screened to assess the performance of the assay in the presence of diverse compounds using the HTS protocol (Fig. 7). The Prestwick collection of compounds was screened at $10 \mu \mathrm{M}$ compound concentration. For this assay, the inhibitor cut-off was defined as $50 \%$ inhibition based on plate controls. Due to solubility limitation of inhibitor, we obtained the min controls on screening plates by leaving the enzyme out of these wells (buffer alone was added). The fluorescence obtained by leaving out the enzyme was the same as that of completely inhibited enzyme (data not shown). We obtained 40 actives (actives were defined as those compounds demonstrating $\geq 50 \%$ inhibition) from this test set screening resulting in a hit rate of $3.6 \%$. The Z'factors of the controls for each of the four plates were all $\geq 0.8$.

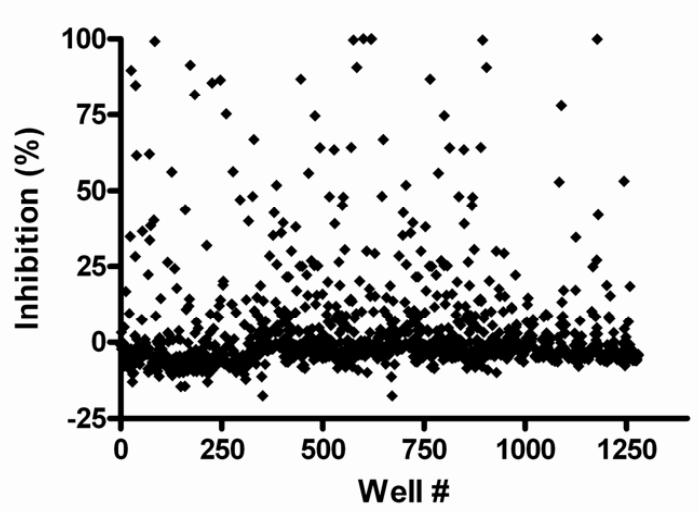

Fig. (7). Screen of the Prestwick collection with the GUS assay. Each point represents a compound. The percent inhibition values were calculated relative to controls on the plates.

A diverse collection of 11,520 small organic molecules were purchased from Asinex Corporation and screened at 10 $\mu \mathrm{M}$ compound concentration for activity in this GUS assay. The plate $Z$ '-factors for the screen were all $\geq 0.82$. A total of 583 actives ( $\geq 50 \%$ inhibition) were obtained for an initial active rate of $5.1 \%$. We sought to limit follow-up confirmatory $\mathrm{IC}_{50}$ determinations to 120 actives, so the active cut-off was raised to $\geq 89 \%$ inhibition to obtain the most potent actives. Of these 120 compounds tested in $\mathrm{IC}_{50}$ assays, 93 of them $(77.5 \%)$ confirmed with an $\mathrm{IC}_{50}$ of $<20 \mu \mathrm{M}$. The Hill slopes generated from such data can be used to triage hits since steep slopes, i.e. significantly greater than 1.0 , can be an indication of compound aggregation which is a nonspecific inhibition mechanism displayed by some compounds [32-33]. Of the 93 confirmed hits, 26 had Hill slopes between 0.8 and 1.2. The potencies of these hits ranged from $50 \mathrm{nM}$ to $4.8 \mu \mathrm{M}$. The structures and activities of 4 of these 26 hits have already been published [29]. Here we show the structures of 8 more novel GUS inhibitors as representative examples of the hits obtained from the screen with $\mathrm{IC}_{50}$ values ranging from 1.7 to $4.8 \mu \mathrm{M}$ (Table 1). The concentration response data used to calculate $\mathrm{IC}_{50}$ values for two compounds are provided as representative curves (Fig. 8). Examination of the structures from Table 1 indicated that 3 of these (compounds 1, 4 and 5) are structurally related to the quinolinone thiourea scaffold previously published [29]. However, compounds 2, 3 and $6-8$ are novel and structurally unique inhibitors of GUS. 
Table 1. Select Confirmed GUS Inhibitors

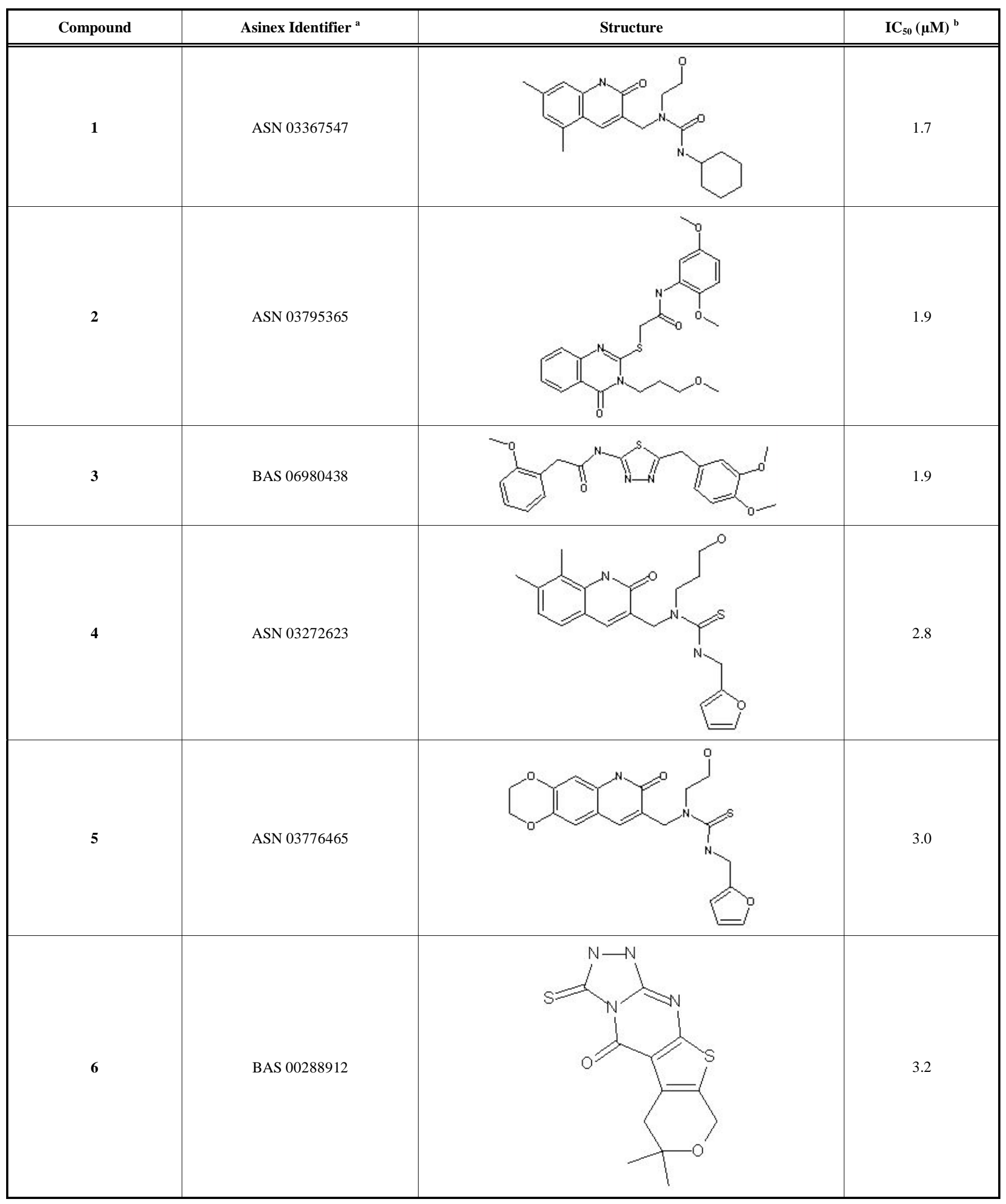


(Table 1). Contd.....

\begin{tabular}{|c|c|c|c|}
\hline Compound & Asinex Identifier a & Structure & $\mathrm{IC}_{50}(\mu \mathrm{M})^{\mathrm{b}}$ \\
\hline 7 & BAS 02056251 & & 4.0 \\
\hline
\end{tabular}

${ }^{\mathrm{a}}$ Chemical identifier number provided by Asinex Corporation

${ }^{\mathrm{b}}$ For $\mathrm{IC}_{50}$ determinations, serial dilutions of compounds were tested starting at a high concentration of $20 \mu \mathrm{M}$. Average IC 50 values ( $\mathrm{n}=3$ ) are shown.

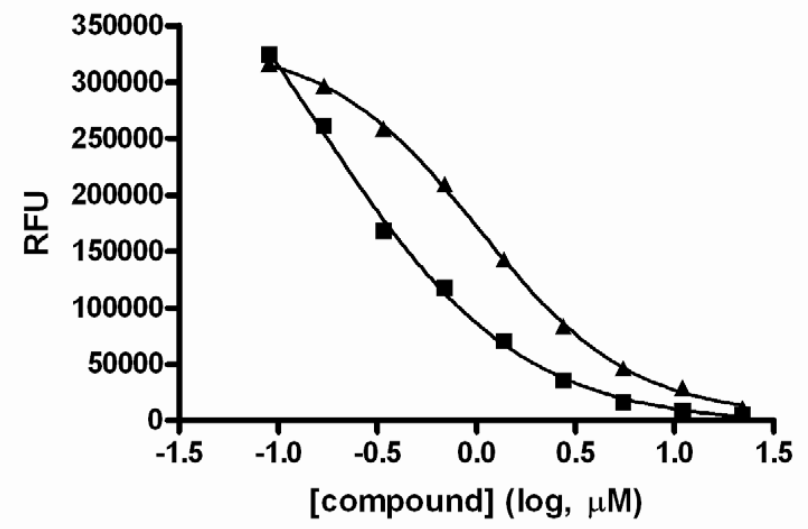

Fig. (8). $I_{50}$ value determination for two screen hits. Compounds were serially diluted in $100 \%$ DMSO then transferred to assay plates for the GUS activity assay. Concentration response curves are shown for two representative hits from the screen.

\section{DISCUSSION}

GUS has been used as a reporter enzyme for many years. It is also the enzyme whose activity is detected in traditional tests for bacterial contamination in water samples. More recently, GUS appears to be responsible for converting the inactive CPT-11 metabolite back to active compound in the intestine. Active SN-38 in the intestine causes the doselimiting diarrhea frequently associated with CPT-11 chemotherapy. These data have led to the hypothesis that small molecules that inhibit bacterial GUS activity may have potential therapeutic value in preventing CPT-11 mediated di- arrhea. Limiting this side-effect may allow increased tolerance for the therapy and/or allow higher doses resulting in better therapeutic outcome.

In this report, we have adapted and validated a high throughput assay to identify inhibitors of GUS activity and used this assay to screen a diverse library of small molecules. The assay signal was shown to increase in a linear fashion with enzyme concentration and time. The assay was also tolerant up to at least $4 \%$ DMSO. As part of the validation, the control inhibitor D-Glucaric acid-1,4-lactone was used to determine $\mathrm{IC}_{50}$ values using the final HTS assay with automation. The $\mathrm{IC}_{50}$ values obtained were consistent between experiments, with the individual values within $12 \%$ of the mean. Whole 384-well plate variability studies using prespotted DMSO plates and automation were done to assess the variability of the assay for detection of inhibitors. The results generated $Z$ '-factors of 0.81 for the inhibition assay window, indicating a robust assay for detection of hits.

As is typical for HTS validation, we screened a small set of compounds (the Prestwick collection) using the automated assay, to assess the performance of the assay in the presence of diverse compounds. This set of four plates resulted in Z'factors of $\geq 0.8$. An active rate of $3.6 \%$ was obtained which predicted in part, the high $5.1 \%$ active rate obtained from the Asinex library screen. The higher than expected active rates may be due the very low enzyme concentration in the assay $(50 \mathrm{pM})$. With no other protein present and only minimal detergent present $(0.01 \%$ Triton X-100), the assay may have been more susceptible to compounds that aggregate or have reactive impurities present. This explanation is supported by the observation that $72 \%$ of the confirmed actives had Hill slopes that were outside the 0.8 to 1.2 range. It has been reported that compounds that form aggregates in aqueous solu- 
tion can non-specifically inhibit purified enzymes, usually generating steep Hill slopes (greater than 1.0) in $\mathrm{IC}_{50}$ value determinations $[32,33]$. The higher active rate obtained in the screen compared to the Prestwick set may be, in part, due to highly related compounds represented in the Asinex library as suggested by the number of quinolinone thiourea compounds identified as hits. Lowering the compound screening concentration, for instance to $1 \mu \mathrm{M}$, or including bovine serum albumin (BSA) in the assay buffer are potential simple solutions that could reduce this hit rate.

From this library screen, at least 26 inhibitors of GUS were identified. The hits from Table 1 represent compounds that are significantly more potent than the control inhibitor. Five of the 8 inhibitors shown here are structurally novel inhibitors of GUS. One scaffold that emerged from the hit list was the quinolinone thiourea scaffold and highly related structures. Four of these compounds were the subject of published follow-up studies that demonstrated that these compounds inhibit GUS in living bacteria with $\mathrm{IC}_{50}$ values ranging from 18 to $1,300 \mathrm{nM}$ without affecting cell viability [29]. They also did not inhibit mammalian GUS. One of these compounds was co-administered to mice receiving CPT-11 with the result that this compound significantly inhibited CPT-11-induced diarrhea. Thus, this GUS assay was successful in finding novel inhibitors of GUS activity for further study.

\section{CONCLUSIONS}

We have developed and validated a fluorescent high throughput assay for the discovery of small molecule inhibitors of GUS activity. Whole plate and $\mathrm{IC}_{50}$ variability studies confirmed the robustness of this assay for HTS. A chemical library was screened resulting in 26 confirmed inhibitors with good Hill slopes $(0.8-1.2)$ with $\mathrm{IC}_{50}$ values ranging from $50 \mathrm{nM}$ to $4.8 \mu \mathrm{M}$. These compounds may be used as starting points for the development of specific small molecule GUS inhibitors as chemical probes. Taken together, the GUS assay described herein is amenable for the discovery of inhibitors of bacterial GUS.

\section{CONFLICT OF INTEREST}

A method of use patent has been filed for the application of some of these compounds for CPT-11-induced toxicity.

\section{ACKNOWLEDGEMENTS}

The authors would like to thank Ginger Smith and Jonathan Z. Sexton for their assistance in performing the high throughput screen and database mining, respectively. This work was supported in part by a grant from the Golden LEAF Foundation, funds from the State of North Carolina and NIH grant CA98468 (M.R.R.).

\section{ABBREVIATIONS}

$$
\begin{array}{ll}
\text { GUS } & =\beta \text {-glucuronidase } \\
\text { DMSO } & =\text { dimethyl sulfoxide } \\
\text { RFU } & =\text { relative fluorescence units } \\
\text { GI } & =\text { gastrointestinal } \\
\text { 4MUG } & =4 \text {-methylumbelliferyl glucuronide } \\
\text { E. coli } & =\text { Escherichia coli } \\
\text { IPTG } & =\text { isopropyl-1-thio-D-galactopyranoside }
\end{array}
$$

\section{REFERENCES}

[1] Wall ME, Wani MC. Camptothecin and taxol: discovery to clinic-thirteenth Bruce F. Cain Memorial Award Lecture. Cancer Res 1995; 55(4): 753-60.

[2] Hsiang YH, Hertzberg R, Hecht S, Liu LF. Camptothecin induces protein-linked DNA breaks via mammalian DNA topoisomerase I. J Biol Chem 1985; 260(27): 14873-8.

[3] Redinbo MR, Champoux JJ, Hol WG. Structural insights into the function of type IB topoisomerases. Curr Opin Struct Biol 1999; 9(1): 29-36.

[4] Pizzolato JF, Saltz LB. The camptothecins. Lancet. 2003; 361(9376): 2235-42.

[5] Pommier Y. Topoisomerase I inhibitors: camptothecins and beyond. Nat Rev Cancer 2006; 6(10): 789-802.

[6] Smith NF, Figg WD, Sparreboom A. Pharmacogenetics of irinotecan metabolism and transport: an update. Toxicol In vitro 2006; 20(2): 163-75.

[7] Ma MK, McLeod HL. Lessons learned from the irinotecan metabolic pathway. Curr Med Chem 2003; 10(1): 41-9.

[8] Mathijssen RHJ, van Alphen RJ, Verweij J, et al. Clinical Pharmacokinetics and Metabolism of Irinotecan (CPT-11). Clin Cancer Res 2001;7(8): 2182-94.

[9] Miley MJ, Zielinska AK, Keenan JE, Bratton SM, RadominskaPandya A, Redinbo MR. Crystal structure of the cofactor-binding domain of the human phase II drug-metabolism enzyme UDPglucuronosyltransferase 2B7. J Mol Biol 2007; 369(2): 498-511.

[10] Nagar S, Blanchard RL. Pharmacogenetics of uridine diphosphoglucuronosyltransferase (UGT) 1A family members and its role in patient response to irinotecan. Drug Metab Rev 2006; 38(3): 393-409.

[11] Stein A, Voigt W, Jordan K. Review: Chemotherapy-induced diarrhea: pathophysiology, frequency and guideline-based management. Ther Adv Med Oncol 2010; 2: 51-63.

[12] Tobin PJ, Dodds HM, Clarke S, Schnitzler M, Rivory LP. The relative contributions of carboxylesterase and beta-glucuronidase in the formation of SN-38 in human colorectal tumours. Oncol rep 2003; 10(6): 1977-9.

[13] Hu ZP, Yang XX, Chan SY, et al. St. John's wort attenuates irinotecan-induced diarrhea via down-regulation of intestinal proinflammatory cytokines and inhibition of intestinal epithelial apoptosis. Toxicol Appl Pharmacol 2006 ; 216(2): 225-37.

[14] Kurita A, Kado S, Matsumoto T, et al. Streptomycin alleviates irinotecan-induced delayed-onset diarrhea in rats by a mechanism other than inhibition of beta-glucuronidase activity in intestinal lumen. Cancer Chemother Pharmacol 2011; 67(1): 201-13.

[15] Flieger D, Klassert C, Hainke S, Keller R, Kleinschmidt R, Fischbach W. Phase II clinical trial for prevention of delayed diarrhea with cholestyramine/levofloxacin in the second-line treatment with irinotecan biweekly in patients with metastatic colorectal carcinoma. Oncology 2007; 72(1-2): 10-6.

[16] Cummings JH, Macfarlane GT. Role of intestinal bacteria in nutrient metabolism. J Parenter Enteral Nutr 1997; 21(6): 357-65.

[17] Guarner F, Malagelada JR. Gut flora in health and disease. Lancet 2003; 361: 512-9.

[18] Cohen SH, Gerding DN, Johnson S, et al. Clinical practice guidelines for Clostridium difficile infection in adults: 2010 update by the society for healthcare epidemiology of America (SHEA) and the infectious diseases society of America (IDSA). Infect Control Hosp Epidemiol 2010; 31(5): 431-55.

[19] Levy SB, Marshall B. Antibacterial resistance worldwide: causes, challenges and responses. Nat Med 2004; 10(12 Suppl): S122-9.

[20] Nord CE, Kager L, Heimdahl A. Impact of antimicrobial agents on the gastrointestinal microflora and the risk of infections. Am J Med 1984; 76(5A): 99-106.

[21] Sears S, McNally P, Bachinski MS, Avery R. Irinotecan (CPT-11) induced colitis: report of a case and review of Food and Drug Administration MEDWATCH reporting. Gastrointest Endosc 1999; 50(6): 841-4.

[22] Settle CD, Wilcox MH. Review article: antibiotic-induced Clostridium difficile infection. Alim Pharmacol Ther 1996; 10(6): 835-41.

[23] Stamp D. Antibiotic therapy may induce cancers in the colon and breasts through a mechanism involving bile acids and colonic bacteria. Med Hypotheses 2004; 63(3): 555-6.

[24] Yang L, Pei Z. Bacteria, inflammation, and colon cancer. World J Gastroenterol 2006; 12(42): 6741-6. 
[25] Basinska A, Florianczyk B. Beta-glucuronidase in physiology and disease. Annales Universitatis Mariae Curie-Sklodowska 2003; 58(2): 386-9.

[26] Farnleitner AH, Hocke L, Beiwl C, Kavka GC, Mach RL. Hydrolysis of 4-methylumbelliferyl-beta-D-glucuronide in differing sample fractions of river waters and its implication for the detection of fecal pollution. Water Res 2002; 36: 975-81.

[27] Fittkau M, Voigt W, Holzhausen H-J, rgen, Schmoll H-J. Saccharic acid 1.4-lactone protects against CPT-11-induced mucosa damage in rats. J Cancer Res Clin Oncol 2004; 130: 388-94.

[28] Russell WM, Klaenhammer TR. Identification and cloning of gusA, encoding a new beta-glucuronidase from Lactobacillus gasseri ADH. Appl Environ Microbiol 2001; 67(3): 1253-61.

[29] Wallace BD, Wang H, Lane KT, et al. Alleviating cancer drug toxicity by inhibiting a bacterial enzyme. Science 2010; 330(6005): $831-5$.
[30] Zhang JH, Chung TD, Oldenburg KR. A Simple Statistical Parameter for Use in Evaluation and Validation of High Throughput Screening Assays. J Biomol Screen 1999; 4(2): 67-73.

[31] Niwa T, Tsuruoka T, Inoue S, Naito Y, Koeda T. A new potent glucuronidase inhibitor, D-glucaro- -lactam derived from nojirimycin. J Biochem 1972; 72(1): 207-11.

[32] Feng BY, Simeonov A, Jadhav A, Babaoglu K, Inglese J, Shoichet $\mathrm{BK}$, et al. A high-throughput screen for aggregation-based inhibition in a large compound library. J Med Chem 2007; 50(10): 2385-90.

[33] McGovern SL, Caselli E, Grigorieff N, Shoichet BK. A common mechanism underlying promiscuous inhibitors from virtual and high-throughput screening. J Med Chem 2002; 45(8): 1712-22.

Received: December 30, 2010

Revised: February 15, 2011

Accepted: March 07, 2011

(C) Ahmad et al.; Licensee Bentham Open.

This is an open access article licensed under the terms of the Creative Commons Attribution Non-Commercial License (http://creativecommons.org/licenses/by-nc/3.0/) which permits unrestricted, non-commercial use, distribution and reproduction in any medium, provided the work is properly cited. 